\title{
Shear bond strength of ceramic laminate veneers to finishing surfaces with different percentages of preserved enamel under a digital guided method
}

\author{
Jiakang Zhu ${ }^{1 \dagger}$, Jing Gao ${ }^{1 \dagger}$, Luming $\mathrm{Jia}^{2}$, Xin Tan ${ }^{1}$, Chenyang Xie ${ }^{3}$ and Haiyang Yu ${ }^{{ }^{*}}$
}

\begin{abstract}
Background: The purpose of this in vitro study was to evaluate the effect of the percentages of preserved enamel on ceramic laminate veneers' (CLVs) shear bond strength (SBS).

Methods: Seventy extracted human maxillary central incisors were scanned and reconstructed into three-dimensional models. The extracted teeth were then embedded and randomly divided into seven groups ( $\mathrm{n}=10$ per group). Based on digital analyses of the three-dimensional models, guided tooth preparation and bonding procedures were performed individually to form seven different percentages (100\%, 80\%, 60\% 50\%, 40\%, 20\% and 0\%) of remaining enamel thickness on the bonding surface. Finally, the SBS test was performed, and the data were statistically analysed by one-way ANOVA with LSD post hoc test $(a=0.05)$.

Results: The complete enamel surface exhibited the highest SBS (19.93 $\pm 4.55 \mathrm{MPa})$, followed by $80 \%$ enamel $(19.03 \pm 3.66 \mathrm{MPa}), 60 \%$ enamel $(18.44 \pm 3.65 \mathrm{MPa}), 50 \%$ enamel $(18.18 \pm 3.41 \mathrm{MPa}), 40 \%$ enamel $(17.83 \pm 3.01 \mathrm{MPa})$ and $20 \%$ enamel $(11.32 \pm 3.42 \mathrm{MPa}$ ) group. The lowest SBS (9.63 $\pm 3.46 \mathrm{MPa})$ was detected in $0 \%$ enamel group. No significant difference was observed among the $40-100 \%$ enamel groups, while the $20 \%$ or $0 \%$ enamel group demonstrated a significantly lower mean SBS than the 40\% enamel group $(p<0.05)$.

Conclusion: The SBS value of CLVs bonded to 100\% enamel on the finishing surfaces (nearly $20 \mathrm{MPa}$ ) was twice that which bonded to $0 \%$ enamel (nearly $10 \mathrm{MPa}$ ). Bonding to $100 \%$ enamel is the most reliable treatment. When dentin exposure is inevitable, enamel should be preserved as much as possible to maintain good bonding. In addition, 40\% of preserved enamel on the bonding surface was the minimal acceptable value to fulfil the requirements of good bonding strength.
\end{abstract}

Keywords: Ceramic laminate veneers, Enamel preservation, Shear bond strength, 3D-printed guide, Finishing surface

\footnotetext{
*Correspondence: YHYmechanics@163.com

${ }^{\dagger}$ Jiakang Zhu and Jing Gao have

contributed equally to this work and should be considered cofirst authors

${ }^{1}$ State Key Laboratory of Oral Diseases, Department of Prosthodontics, National Clinical Research Center for Oral Diseases, West China Hospital of Stomatology, Sichuan University, 14 Renmin South Road, 3rd section, Chengdu 610041, Sichuan, People's Republic of China

Full list of author information is available at the end of the article
}

\section{Background}

Laminate veneers are clinically indicated for various aesthetic reasons, leading to a more minimally invasive treatment by allowing more tooth structure to be preserved [1]. However, laminate veneers are also prone to failure because of the higher technical sensitivity $[2,3]$. Debonding is one of the most common reasons for the failure of laminate veneers, and there are many factors 
contributing to debonding, such as the types of adhesive system and resin cement, tooth preparation depths and types, and functional and parafunctional activities, etc. $[4,5]$ As one of the most important factors affecting debonding, the depths of preparation influence the percentage of enamel surface on the adhesive surfaces, which is crucial for maintaining enough bonding strength of laminate veneers. Previous in vitro studies have confirmed that the shear bond strength (SBS) of ceramic to enamel was higher than to dentin [6,7], and longitudinal studies have shown that the survival rate of laminate veneers after 10 years is more than $90 \%$ if the enamel bonding surface is sufficient $[8,9]$. However, the effects of enamel preservation on the shear bond strength (SBS) of laminate veneers have not been clearly quantified.

Computer-guided tooth preparation is developed from the technologies of dental scanners, computeraided design (CAD) software, and computer-aided manufacturing (CAM) software. Guided tooth preparation promotes precisely controlled depths of preparation, and digital spatial analysis facilitates the completion intra-enamel preparation [10-12]. Nevertheless, in regard to discoloured or misaligned teeth, more reduction of tooth structure may be required to improve the aesthetic result, causing inevitable dentin exposure [13]. For laminate veneers, what is the acceptable range of dentin exposure? Öztürk and others [6] indicated that compared with bonding to enamel, the bonding strength of ceramic to enamel-dentine complex had no significant reduction. However, it is worth noting that the enamel/dentin ratio was estimated to be approximately $1: 1$ in that study without accurate calculation. Some dentin exposure was allowed in the treatment [14], but further research about the critical value was deficient.

The aims of this study were to create different percentages of preserved enamel amount on finishing surfaces through a digital guided method and to compare the SBS of the different percentages of preserved enamel. In addition, it is expected to preliminarily explore the acceptable extent of dentin exposure for CLVs. The null hypothesis was that there is no association between the percentages of preserved enamel amount and SBS values for CLVs on maxillary central incisors.

\section{Methods}

\section{Specimen collection}

The protocol of this study was approved by the Ethics Committee (Approval Number: WCHSIRBD-2019-122). Seventy non-carious human maxillary central incisors were stored in $0.5 \%$ chloramine in water at $4{ }^{\circ} \mathrm{C}$ and used within 1 month after extraction. Figure 1 illustrates the workflow diagram of this study.

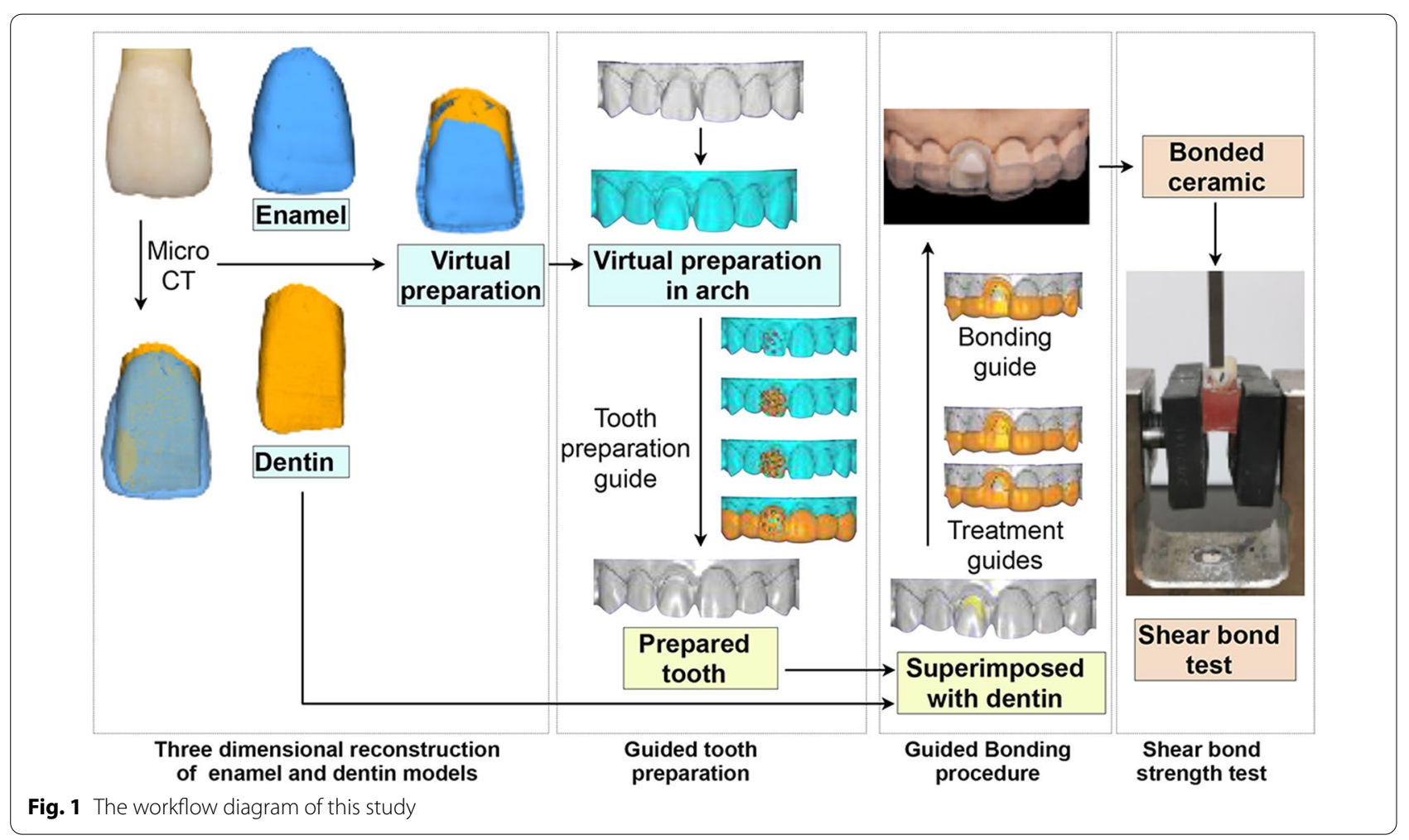




\section{Design of tooth preparation guides}

The extracted teeth were scanned by micro-computed tomography (micro-CT) ( $\mu \mathrm{CT}$ 50; SCANCO Medical AG, Bassersdorf, Switzerland) (scanning parameters: $80 \mathrm{kV}, 500 \mu \mathrm{A}, 19.64 \mu \mathrm{m}$, and $800 \mathrm{~ms}$ ) to reconstruct their three-dimensional (3D) enamel and dentin models in reverse engineering software (Mimics 17.0; Materialise, Leuven, Belgium). All teeth were mounted in dental gypsum to simulate the dental arch, which was scanned by an intraoral scanner (TRIOS Colour Pod; 3Shape, Copenhagen, Denmark) and saved in a standard tessellation language (STL) format. The arch models were randomly divided into seven groups $(\mathrm{n}=10$ each). The analysis based on the power.anova.test function of the stats package (3.6.2) showed that using 10 specimens per group would give a needed power. And this is also consistent with the number of specimens used in the recently published literatures on shear bond strength test [15-17].

Each digital arch model was imported into Materialise software (Magics 23; Materialise, Leuven, Belgium), and virtual preparation was conducted on the sample tooth, as described by Gao et al. [10]. Briefly, the labial surface was selected and shifted inward by using the "Offset" tool. Different depths of preparation were designed to form different percentages of preserved enamel on the finishing surfaces in seven groups. To convert the depths of virtual preparation to definitive preparation, tooth preparation guides were designed based on virtual preparation, as described by Liu et al. [12].

\section{Guided tooth preparation}

Guided tooth preparation was conducted by one operator who was unaware of the experimental aims and assessment criteria of the study (Fig. 2). Briefly, depth-guiding dimples were created with the aid of a tooth preparation guide by using a calibrated bur (HX-1; Gaofeng, Wuxi, China). Then, the dimples were marked with a pencil, the remaining tooth tissues between the dimples were removed and tooth surfaces were finished by a tapered carbide bur (HX-2; Gaofeng, Wuxi, China). Following this process, the depth of preparation was consistent with that of the virtual preparation, which was designed to form a specific enamel/dentin ratio on the tooth surface. The prepared teeth were scanned and saved as "STL" files, and the specimens were stored in artificial saliva.

\section{Fabrication of ceramic laminate veneers}

Lithium disilicate ceramic mini veneers (IPS e. $\max$ Press; Ivoclar Vivadent, Schaan, Liechtenstein) were designed and milled by a chairside CAD/CAM machine (Cameo N4; Aidite, Qinhuangdao, China). To simulate the shape of the veneer, the adhesive surface was in the form of a Reuleaux triangle whose sides were replaced with circular arcs with a curve of constant width (Fig. 3g). The adhesive surfaces of all the specimens were finished with 600-grit abrasive paper to create a uniform surface. All specimens were cleaned with $75 \%$ alcohol, washed in deionized water by ultrasonication for $5 \mathrm{~min}$, and dried at room temperature.

\section{Design of the treatment and adhesive guides}

The digitally prepared tooth was superimposed with its enamel and dentin models in dental CAM software (Exocad 2018; Exocad GmbH, Darmstadt, Germany), where the enamel and dentin surfaces were presented on the tooth surface. Then, the same Reuleaux triangle was shifted on to the finishing surface until the percentage of preserved enamel in the area of the Reuleaux triangle fulfilled the requirement. Thus, the adhesive surface of the tooth, as the shape of the veneer, was designed to form a respective percentage of preserved enamel in each group (Table 1). Accordingly, the treatment guides were designed to locate the enamel and dentin surfaces, and the bonding guides were designed to locate the adhesive surface (Fig. 3). All guides were fabricated using the 3D printer (ProJet MJP 3600; 3D Systems, Rock Hill, SC).

\section{Guided bonding procedure}

Figure 4 shows the bonding procedure in the present study. Enamel and dentin surfaces were treated under the treatment guides following the treatment procedures in Table 2. Then, the bonding guide was seated, and adhesion was performed according to the instructions of the manufacturers (Rely X veneer; $3 \mathrm{M}$, Sao Paulo, MN). Finally, the bonding guide was cut with a tapered fissure bur and gently removed. A pressure control $(100 \mathrm{~N})$ was conducted to standardize the bonding procedure and to withstand the debonding forces from the removal of the guide.

\section{The SBS test}

The sample tooth was removed from the arch and vertically embedded in acrylic resin (polymethyl methacrylate; Nissin, Tokyo, Japan) to place the cementoenamel junction (CEJ) plane under $2.5 \mathrm{~mm} \pm 0.5 \mathrm{~mm}$ from the top platform of the resin surface. The specimens were stored in artificial saliva for $24 \mathrm{~h}$ at $37{ }^{\circ} \mathrm{C}$ and were thermocycled between 5 and $55^{\circ} \mathrm{C}$ in deionized water for 5000 cycles. The test head was sheared from incisal to cervical. The SBS test was conducted with a universal mechanical testing machine (INSTRON-5565; INSTRON, Boston, MA) at a crosshead speed of $0.5 \mathrm{~mm} / \mathrm{s}$ until failure on all samples. The test results were recorded in MPa. 

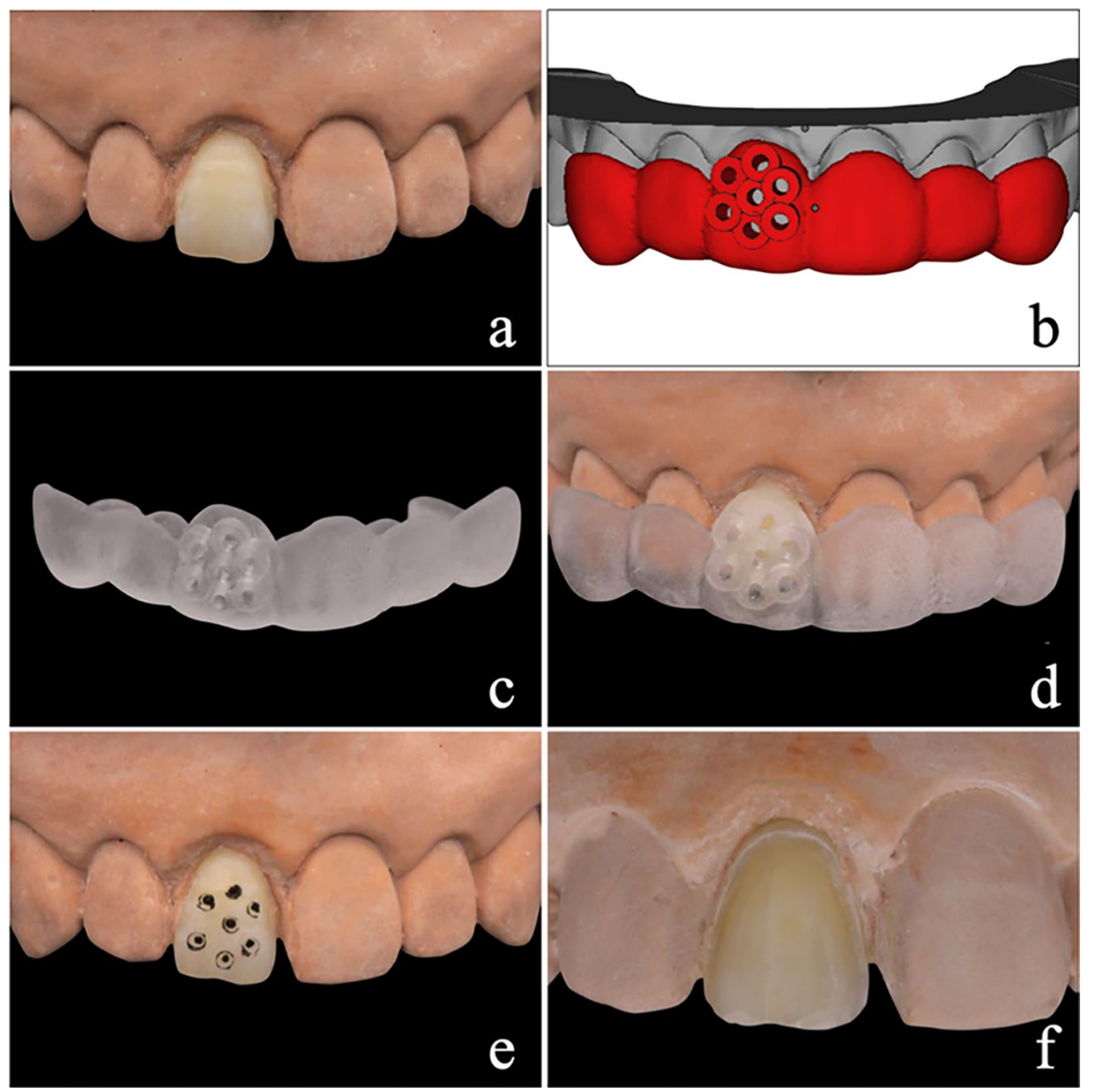

Fig. 2 Guided tooth preparation. a Embedded tooth. $\mathbf{b}$ Design of tooth preparation guide. $\mathbf{c}$ Tooth preparation guide. $\mathbf{d}$ Location of tooth preparation guide. e Depth control holes. f Prepared tooth surface

\section{Failure analysis and scanning electron microscopy (SEM) examination}

After the SBS test, all specimens were examined under a stereomicroscope (BX51M; OLYMPUS, Tokyo, Japan) at a magnification of $50 \times$ to determine the fracture mode. Representative specimens were selected for fractographic examination by SEM (Inspect F50; FEI, Hillsboro, OR) at $5000 \times$ magnification. Possible failure modes that were similar to the classification by Scherrer and others [18] and Öztürk and others [6] were classified as follows:

1. Adhesive failure: fracture between the ceramic and tooth surface within the adhesive interface,

2. Mixed failure: are cohesive failures (less than $40 \%$ of the interface) in the tooth structure and adhesive interface combined with adhesive failure between the ceramic and tooth,

3. Cohesive failure: internal fracture of the tooth structure or adhesive interface (more than $40 \%$ of the bonding interface).

4. To quantify the proportion of internal fractures, Image J software was used to estimate the percentage of internal fracture area.

\section{Statistical analysis}

Numerical (quantitative) data were presented as the mean and standard deviation values. Categorical (qualitative) data were presented as frequencies and percentages. One-way analysis of variance and the least significant 


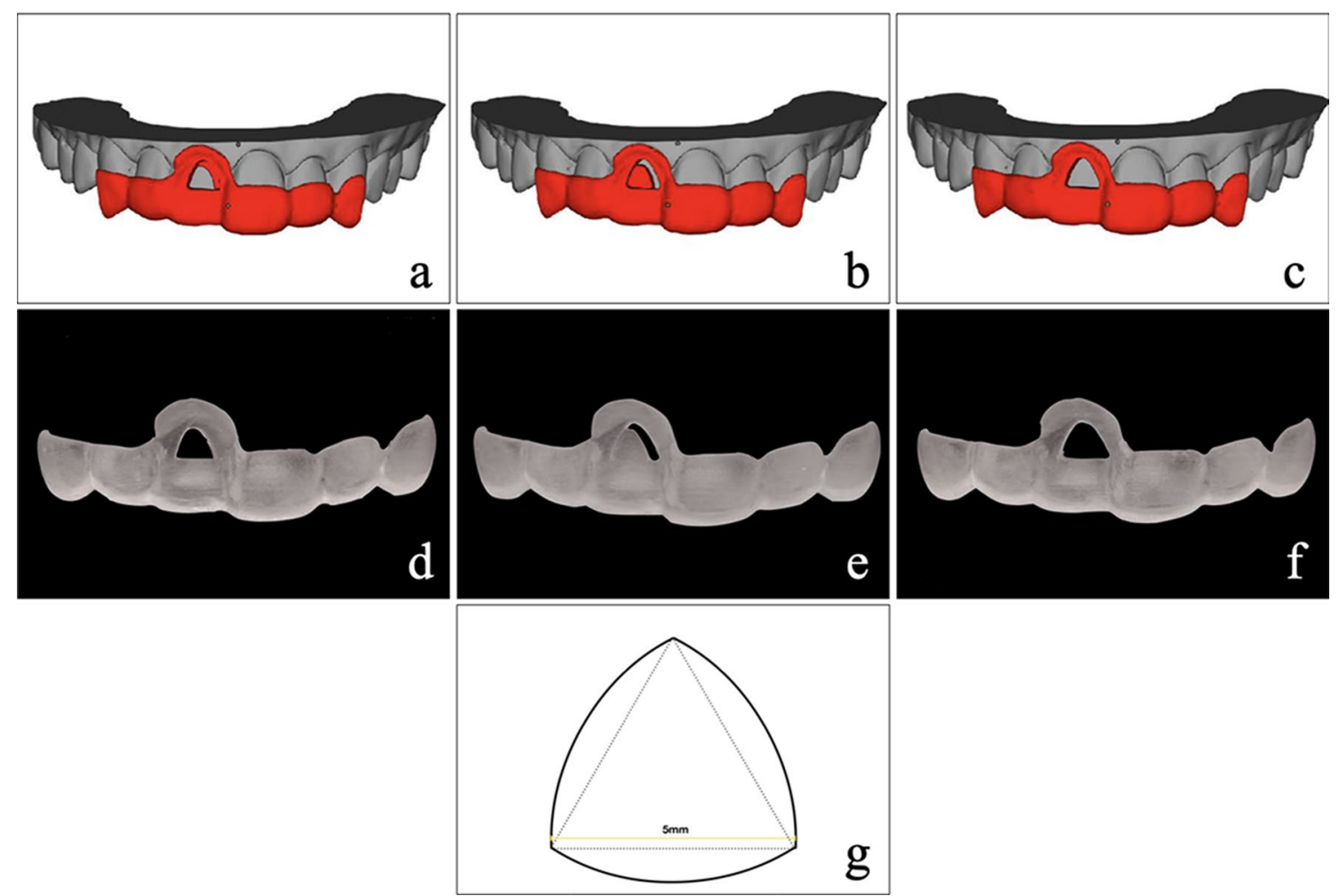

Fig. 3 Adhesive guides. a Design of enamel treatment guide. $\mathbf{b}$ Design of dentine treatment guide. $\mathbf{c}$ Design of bonding guide. $\mathbf{d}$ Enamel treatment guide. $\mathbf{e}$ Dentine treatment guide. $\mathbf{f}$ Bonding guide. $\mathbf{g}$ The form of adhesive surface: a reuleaux triangle

Table 1 The percentages of preserved enamel on the selected adhesive surface in seven gropes

\begin{tabular}{llllllll}
\hline Groups & G1 & G2 & G3 & G4 & G5 & G6 & G7 \\
\hline The percentages of preserved enamel & 100 & 80 & 60 & 50 & 40 & 20 & 0 \\
\hline
\end{tabular}

difference (LSD) test were used to calculate statistics on the fracture strength among different groups. Fisher's exact test was used to compare the failure modes of the four groups. The test standard was bilateral $\alpha=0.05$. The significance level was set at $p<0.05$. Statistical analysis was performed with SPSS software (SPSS 26.0; SPSS, Chicago, IL).

\section{Results}

Figure 5 shows the selected adhesive surfaces from different groups. The finishing surfaces were reconstructed by merging the 3D dentin model and the prepared tooth model. As shown in Fig. 5a-g, the percentages of preserved enamel amount on selected adhesive surfaces of the seven groups were $100 \%, 80 \%, 60 \%, 50 \%, 40 \%, 20 \%$ and $0 \%$.

Figure 6 presents the SBS values of different percentages of preserved enamel. Among the 7 groups, G1 exhibited the highest mean SBS value $(19.93 \pm 4.55 \mathrm{MPa})$, followed by G2 $(19.03 \pm 3.66 \mathrm{MPa})$, G3 (18.44 $\pm 3.65 \mathrm{MPa}), \mathrm{G} 4(18.18 \pm 3.41 \mathrm{MPa})$ and G5(17.83 $\pm 3.01 \mathrm{MPa})$ groups. No significant difference was observed among the G1, G2, G3, G4 and G5 groups $(p>0.05)$, but $\mathrm{G} 5$ demonstrated a significantly higher SBS value thanG6 $(11.32 \pm 3.42 \mathrm{MPa})(p<0.05)$. G7 showed the lowest mean SBS value $(9.63 \pm 3.46 \mathrm{MPa})$ among these groups, but there was no significant difference between the G6 and G7 groups $(p>0.05)$.

The failure mode distribution in the seven groups is presented in Fig. 7. The most frequently experienced failure type was adhesive failures in all groups, and the G7 group was the most frequent, with 10 failures $(100 \%)$. Mixed failures were observed in 6 groups except for G7, among which the G1 and G2 groups had the most failures with $6(60 \%)$. Cohesive failure was 

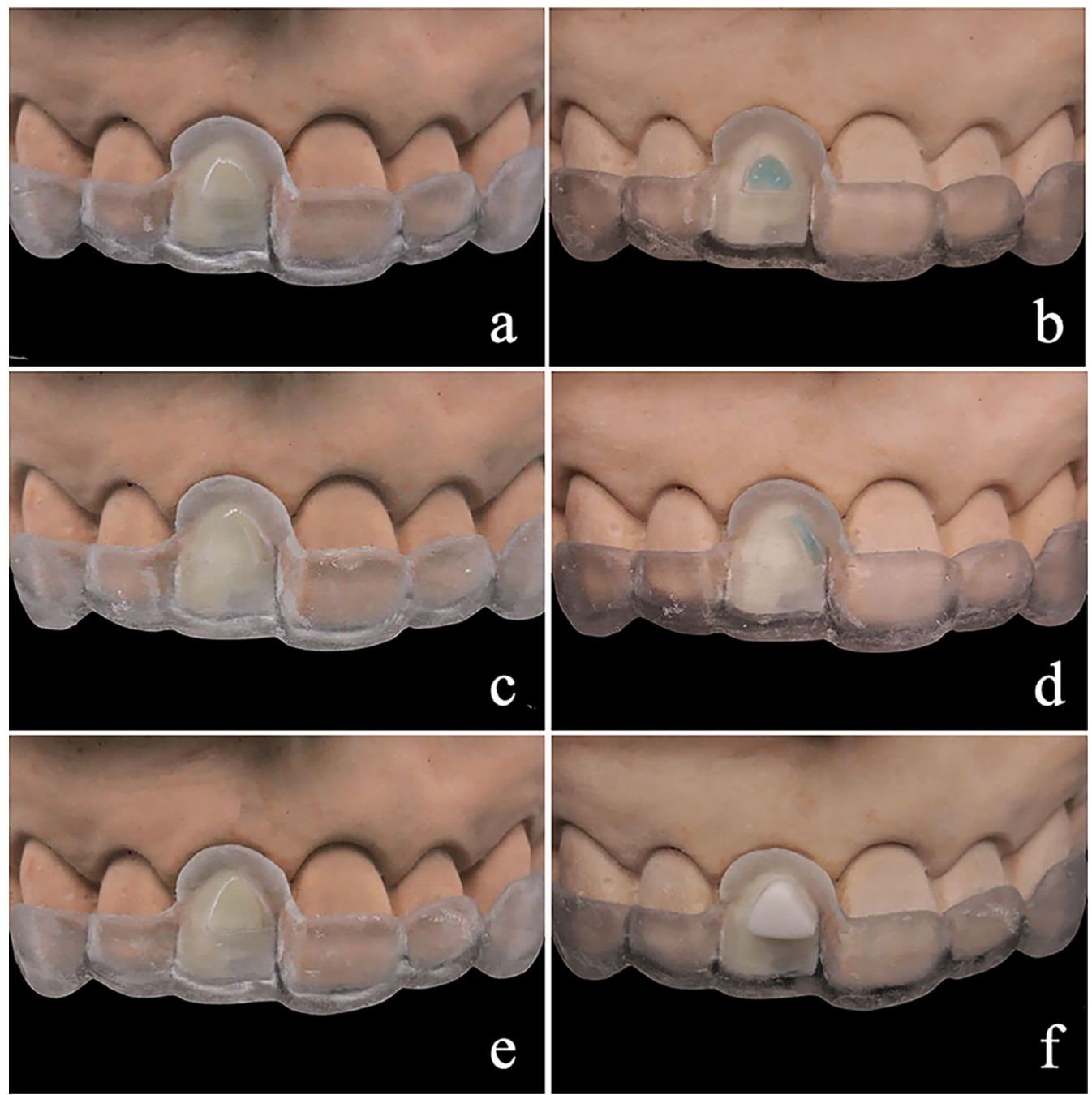

Fig. 4 Guided bonding procedure. a Location of enamel treatment guide. b Enamel treatment under guide with $37 \% \mathrm{H}_{3} \mathrm{PO}_{4}$ for 30 s. c Location of dentine treatment guide. $\mathbf{d}$ Dentine treatment under guide with $37 \% \mathrm{H}_{3} \mathrm{PO}_{4}$ for $3 \mathrm{~s}$. e Adhesive surface treatment under bonding guide with single bond for $10 \mathrm{~s}$. f Ceramic bonded on the tooth

Table 2 Surface procedures of the teeth and ceramics

\begin{tabular}{ll}
\hline Surface & Surface treatments \\
\hline Porcelain & $5 \% \mathrm{HF}$ for $60 \mathrm{~s}$; primer for $60 \mathrm{~s}$ \\
Enamel & $37 \% \mathrm{H}_{3} \mathrm{PO}_{4}$ for $30 \mathrm{~s}$; single bond for $10 \mathrm{~s}$ \\
Dentin & $37 \% \mathrm{H}_{3} \mathrm{PO}_{4}$ for $3 \mathrm{~s}$; single bond for $10 \mathrm{~s}$ \\
\hline
\end{tabular}

H3PO4: phosphoric acid (total etch, ivoclar vivadent, schaan, liechtenstein). HF: hydroffluoric acid (vita ceramics etch, VITA zahnfabrik, Bad Sackingen, Germany)

found in the G1, G2, G3 and G4 groups; among them, the $\mathrm{G1}$ group had the greatest number $(3 ; 30 \%)$.

Figure 8 shows SEM micrographs of the tooth surfaces after the shear bond strength test. Figure 8 a illustrates the cohesive failure of a specimen from the G4 group. This type of failure indicates that internal fracture of the tooth structure or adhesive interface occurred and the area was more than $40 \%$ of the bonding interface. Figure $8 \mathrm{~b}$ is an example of a mixed failure from the G5 group. Mixed failure indicates a mixture of cohesive failure (less than $40 \%$ of the interface) and adhesive failure within the same fracture surface. Figure 8c shows representative micrographs of adhesive failure. The fracture occurred in the tooth structure or adhesive interface.

\section{Discussion}

This study was the first to achieve quantitative classification of the percentages of preserved enamel amount on the finishing surface through a digital guided method. Tooth preparation guides were designed to transfer the reduction depths of virtual preparations to definitive 


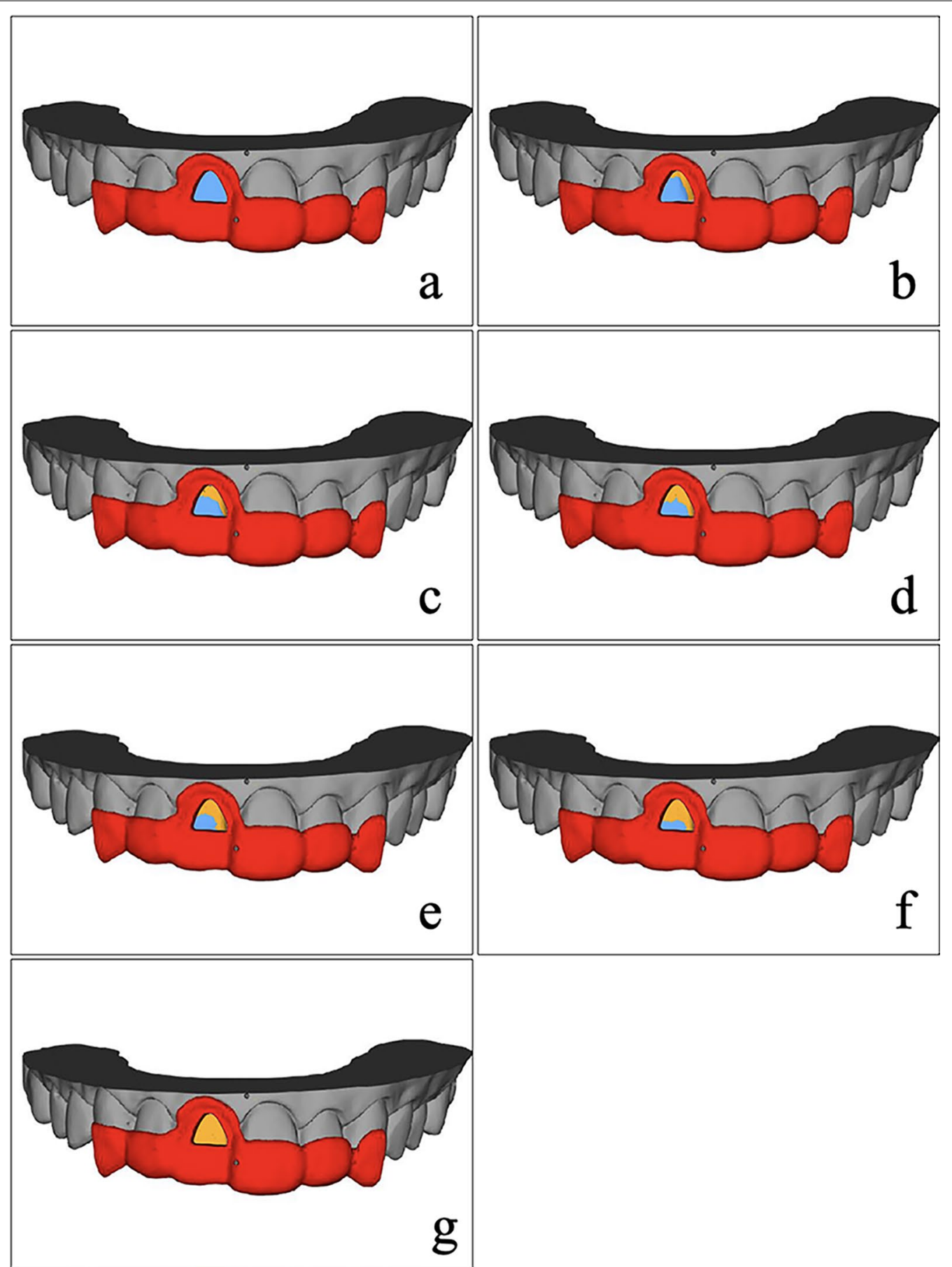

Fig. 5 The adhesive surfaces from seven groups. a 100\% enamel presented. b $80 \%$ enamel presented. c $60 \%$ enamel presented, d $50 \%$ enamel presented, e $40 \%$ enamel presented. f $20 \%$ enamel presented, $\mathbf{g} 0 \%$ enamel presented

preparation [19-21]. Enamel substrate was presented by the superimposition of the prepared tooth and its dentin models [22, 23], by which the adhesive guides could be designed to locate the enamel and dentin adhesive surface. With the aid of the surface treatment guides, the enamel and dentin surfaces could be presented and treated separately without impacting each other. The adhesive surface of mini veneers was designed as a Reuleaux triangle to simulate the shape of veneers in the clinic, based on a method comparable to the ISO 29022 


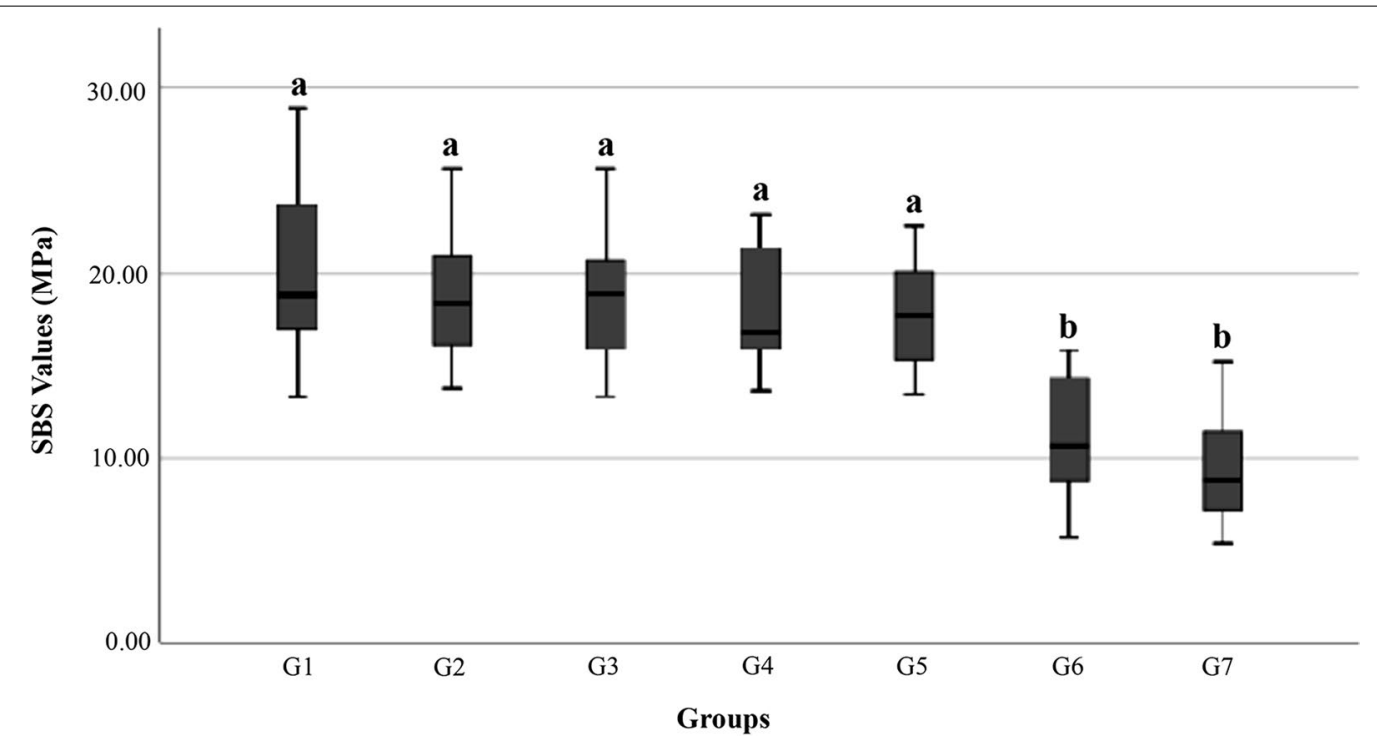

Fig. 6 The shear bond strength test in seven groups. Different lower-case letters indicate the significant statistical difference $(p<0.05)$

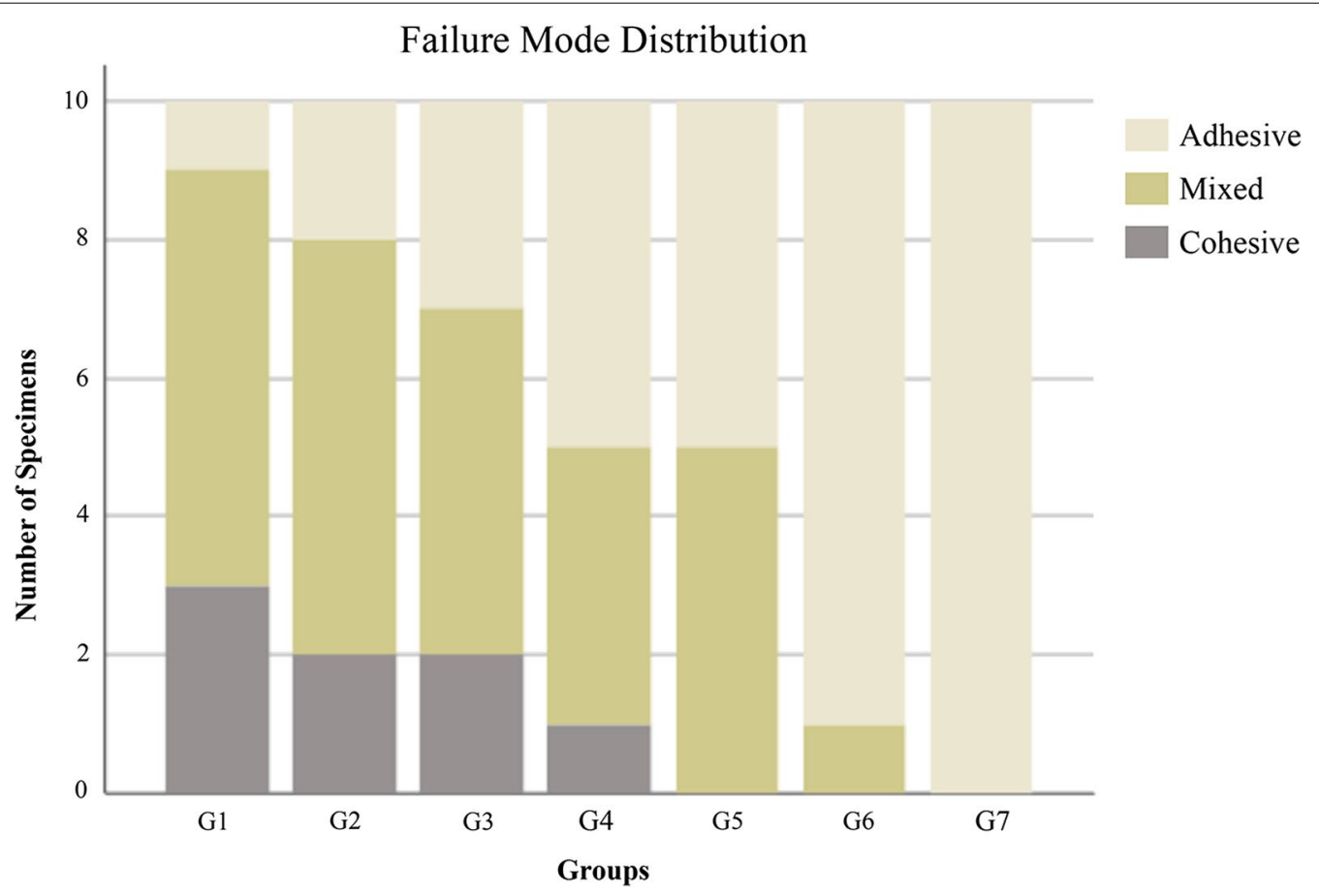

Fig. 7 Distribution of adhesive failure types among seven groups

shear test [24]. With the help of the digital workflow, seven levels of the percentages of preserved enamel amount on the finishing surface were established and precisely transferred to the teeth specimens.

In this study, the percentages of preserved enamel amount had a significant effect on the shear bond strength, and the results rejected the null hypothesis that there was no association between the percentages of preserved enamel and SBS values for CLVs on maxillary central incisors. In this research, we discovered that the $100 \%$ enamel surface group showed the highest mean SBS value among the 7 groups, which correlated with previous reports [6]. The SBS value of CLVs bonded to 100\% enamel on the finishing surfaces was nearly $20 \mathrm{MPa}$, 

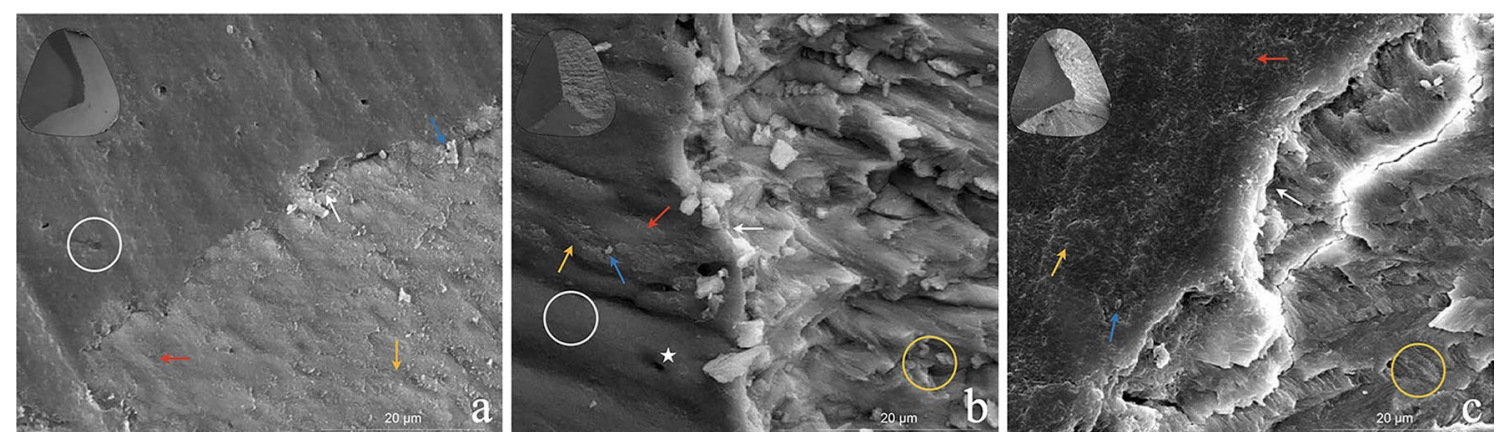

Fig. 8 SEM micrographs of fractured specimens. a Cohesive failure from the G4 group. b Mixed failure from the G4 group. c Adhesive failure from the G4 group. white circle: dentin; yellow circle: enamel; yellow arrow: residual adhesive; White arrow: fracture interface; Red arrow: Internal structure of adhesive; blue arrow: filler content of adhesive; $\times 50$ fracture interface in black circle at upper left corner

which was twice that bonded to dentin. The results indicated that bonding to complete enamel is still the most reliable treatment. The degree of mineralization in enamel is higher than that in dentin. The honeycomb structure formed after demineralization of hydroxyapatite is favourable for the formation of resin protrusion in enamel. However, there are more organic components in dentin, and dentin tubules contain much water. Inappropriate acid-etching and drying can lead to the collapse of the collagen fibre network, which has a great impact on dentin bonding $[25,26]$. Collectively, tooth preparation for CLVs should be controlled in enamel as much as possible to ensure the highest SBS value.

The study showed that there was no significant difference in bond strength among the $40-100 \%$ enamel groups, indicating that a small amount of dentin exposure is acceptable during preparation for CLVs in clinics. We also observed that there was no significant difference in bond strength between the $20 \%$ and $0 \%$ enamel groups. Thus, extensive dentine exposure should be avoided. Notably, bonding to $40 \%$ enamel demonstrated significantly higher bond strength than bonding to $20 \%$ enamel. Previous studies have reported that more debonding of CLVs would be presented when less than $50 \%$ of enamel was preserved $[13,27,28]$. However, the value $(50 \%)$ was just a ballpark estimate, lacking relatively accurate calculation. It has been reported that the SBS value of adhesives to dentin should be at least $17 \mathrm{MPa}$, while that of enamel should be $20 \mathrm{MPa}$ to adequately compensate for the stresses caused by polymerization shrinkage [ 6 , 29]. Owing to the different experimental conditions and methods, in this study, the mean shear bond strength values of the $40-100 \%$ enamel groups were above $17 \mathrm{MPa}$. However, mean shear bond strength values of $20 \%$ and $0 \%$ groups were well below $17 \mathrm{MPa}$. Considering all these data and our results, it is reasonable to suggest that CLVs could be applied only when at least $40 \%$ of enamel is preserved on the finishing surface after preparation to guarantee good bonding.

In addition, cohesive failure is only observed in bonding to $50-100 \%$ enamel groups. Cohesive failure is attributed to the adhesive bond strength exceeding the intrinsic strength of the tooth, so this type of failure indicates increased bond strength between the resin cement and teeth [30,31]. This is consistent with the higher SBS values in these four groups. However, structural features of the extracted teeth, increased fragility of teeth after a long storage time, and nonuniform distribution of stress, are also predisposing factors for cohesive failures [32]. Adhesive failure was the most frequent fracture type, bonding to $20 \%$ enamel presented significantly more adhesive failures than $40 \%$ enamel, and bonding to $0 \%$ enamel presented only adhesive failures. This result is in accordance with previous investigations that showed that when the shear bond strength values of resin cement to the dentin surface are lower, adhesive bond failure is more likely to occur [33,34]. Taken together, these results confirmed the critical role of $40 \%$ enamel preservation in tooth preparation for CLVs, which demonstrates significantly higher bond strength than bonding to $20 \%$ enamel.

Due to the limitation of the SBS test, the specimen of CLVs was designed as a mini veneer like Reuleaux triangle, which could not completely simulate the actual clinical situation. In addition, experimental conditions were different from clinical treatment. However, the study still had certain guiding significance for clinicians. For CLVs, tooth preparation should be finished in enamel as far as possible to reduce the risk of failure. In regard to inevitable dentin exposure [35], the percentages of enamel adhesive surfaces should be more than $40 \%$ to guarantee the longevity of CLVs, otherwise, other treatments such as full crowns, may be considered [36, 37]. In addition, it has been suggested that the preparation margins should be in sound enamel for CLVs to improve the bonding 
strength between teeth and restorations as well as reduce the incidence of secondary caries caused by microleakage $[38,39]$.

\section{Conclusions}

Within the limitations of this study, the following conclusions can be addressed:

1. Complete intra-enamel preparation is the most optimal for CLVs by providing the highest SBS values. Enamel preservation of $40 \%$ is the essential threshold value during tooth preparation for CLVs to ensure enough bond strength of CLVs.

2. The digital guided method promotes a more reliable process for the enamel preservation. Tooth preparation guide improves the accuracy of reduction depth for veneer preparation, and adhesive guides allow the location of enamel, and dentin on the adhesive surfaces.

\begin{abstract}
Abbreviations
CLVs: Ceramic laminate veneers; SBS: Shear bond strength; CAD: Computeraided design; CAM: Computer-aided manufacturing; 3D: Three-dimensional; STL: Standard tessellation language; CEJ: Cemento-enamel junction; SEM: Scanning electron microscopy.
\end{abstract}

\section{Acknowledgements}

Not applicable.

\section{Authors' contributions}

Study design: HY. Experiments and data analyses: JZ, JG, LJ, CX. Drafted the manuscript: JZ, JG, XT. Reviewed the manuscript: HY. All authors have read and approved the final manuscript.

\section{Funding}

This study was supported by Sichuan Science and Technology Program, grant \# 2020YFS0040. The funding of this study had no influence in the process of study design, data collection, analysis and interpretation of our results and writing the manuscript.

\section{Availability of data and materials}

The data of this study are available from corresponding author on reasonable request.

\section{Declarations}

Ethics approval and consent to participate

The study protocol was approved by the Institutional Review Board (IRB) of the West China Hospital of Stomatology (WCHSIRB-D-2019-122). Written informed consent was obtained from each tooth donor.

\section{Consent for publication}

Not applicable.

\section{Competing interests}

The authors declare that they have no competing interest.

\section{Author details}

${ }^{1}$ State Key Laboratory of Oral Diseases, Department of Prosthodontics, National Clinical Research Center for Oral Diseases, West China Hospital of Stomatology, Sichuan University, 14 Renmin South Road, 3rd section, Chengdu 610041, Sichuan, People's Republic of China. ${ }^{2}$ BYBO Dental Hospital, Beijing, People's Republic of China. ${ }^{3}$ Department of Dental Technology, West China Hospital of Stomatology, Sichuan University, Chengdu, People's Republic of China.

Received: 29 Auqust 2021 Accepted: 28 December 2021

Published online: 07 January 2022

\section{References}

1. Edelhoff $D$, Sorensen JA. Tooth structure removal associated with various preparation designs for anterior teeth. J Prosthet Dent. 2002;87(5):503-9.

2. AlJazairy $\mathrm{YH}$. Survival rates for porcelain laminate veneers: a systematic review. Eur J Dent. 2020;15(02):360-8.

3. Kursoglu P, Motro PF. An alternative method for cementing laminate restorations with a micropulse toothbrush. J Prosthet Dent. 2014;112(6):1595-6.

4. Granell-Ruiz M, Fons-Font A, Labaig-Rueda C, Martínez-González A, Román-Rodríguez JL, Solá-Ruiz MF. A clinical longitudinal study 323 porcelain laminate veneers: period of study from 3 to 11 years. Med Oral Patol Oral Cir Bucal. 2010;15(3):e531-537.

5. Morimoto S, Albanesi RB, Sesma N, Agra CM, Braga MM. Main clinical outcomes of feldspathic porcelain and glass-ceramic laminate veneers: a systematic review and meta-analysis of survival and complication rates. Int J Prosthodont. 2016;29(1):38-49.

6. Öztürk E, Bolay Ş, Hickel R, llie N. Shear bond strength of porcelain laminate veneers to enamel, dentine and enamel-dentine complex bonded with different adhesive luting systems. J Dent. 2013;41(2):97-105.

7. Türk AG, Cal E, Unal S, Güneri P, Ulusoy M, Ozden M. Glass-ceramics bonding in geriatric patients: comparison with young teeth. Gerodontology. 2017;34(1):49-56.

8. Alenezi A, Alsweed M, Alsidrani S, Chrcanovic BR. Long-term survival and complication rates of porcelain laminate veneers in clinical studies: a systematic review. J Clin Med. 2021;10(5):1074.

9. Burke FJ. Survival rates for porcelain laminate veneers with special reference to the effect of preparation in dentin: a literature review. J Esthet Restor Dent. 2012;24(4):257-65.

10. Gao J, Li J, Liu C, Fan L, Yu J, Yu H. A stereolithographic template for computer-assisted teeth preparation in dental esthetic ceramic veneer treatment. J Esthet Restor Dent. 2020;32(8):763-9.

11. Yu H, Zhao Y, Li J, Luo T, Gao J, Liu H, Liu W, Liu F, Zhao K, Liu F, et al. Minimal invasive microscopic tooth preparation in esthetic restoration: a specialist consensus. Int J Oral Sci. 2019;11(3):31.

12. Liu C, Guo J, Gao J, Yu H. Computer-assisted tooth preparation template and predesigned restoration: a digital workflow. Int J Comput Dent. 2020;23(4):351-62.

13. Gurel G, Sesma N, Calamita MA, Coachman C, Morimoto S. Influence of enamel preservation on failure rates of porcelain laminate veneers. Int J Periodontics Restorative Dent. 2013;33(1):31-9.

14. Oztürk E, Bolay S. Survival of porcelain laminate veneers with different degrees of dentin exposure: 2-year clinical results. J Adhes Dent. 2014;16(5):481-9.

15. Alberto Jurado C, Kaleinikova Z, Tsujimoto A. Comparison of fracture resistance for chairside CAD/CAM lithium disilicate crowns and overlays with different designs. J Prosthodont. 2021. https://doi.org/10.1111/jopr. 13411.

16. Celik EU, Ergücü Z, Türkün LS, Türkün M. Shear bond strength of different adhesives to Er:YAG laser-prepared dentin. J Adhes Dent. 2006:8(5):319-25.

17. Yi YA, Ahn JS, Park YJ, Jun SH, Lee IB, Cho BH, Son HH, Seo DG. The effect of sandblasting and different primers on shear bond strength between yttria-tetragonal zirconia polycrystal ceramic and a self-adhesive resin cement. Oper Dent. 2015;40(1):63-71.

18. Scherrer SS, Cesar PF, Swain MV. Direct comparison of the bond strength results of the different test methods: a critical literature review. Dent Mater. 2010;26(2):e78-93.

19. Lee H, Fehmer V, Kwon KR, Burkhardt F, Pae A, Sailer I. Virtual diagnostics and guided tooth preparation for the minimally invasive rehabilitation of 
a patient with extensive tooth wear: a validation of a digital workflow. J Prosthet Dent. 2020;123(1):20-6.

20. Sadid-Zadeh R, Guha U, Arany H. Use of virtual comparison software for preparation depth assessment in a dental technique skills course. J Dent Educ. 2018;82(8):891-7.

21. Li ZY, Bai HF, Zhao YJ, Wang Y, Ye HQ, Sun YC. 3D evaluation of accuracy of tooth preparation for laminate veneers assisted by rigid constraint guides printed by selective laser melting. Chin J Dent Res. 2020;23(3):183-9.

22. Al-Fouzan AF, Tashkandi EA. Volumetric measurements of removed tooth structure associated with various preparation designs. Int J Prosthodont. 2013;26(6):545-8.

23. Wang P, Sun F, Yu Q, Wu G. Three-dimensional analysis of the relationship between the structure of maxillary central incisor and the preparation of dental all-ceramic. PLoS ONE. 2018;13(12):e0209791.

24. Hu M, Weiger R, Fischer J. Comparison of two test designs for evaluating the shear bond strength of resin composite cements. Dent Mater. 2016;32(2):223-32.

25. Carrilho MR, Geraldeli S, Tay F, de Goes MF, Carvalho RM, Tjäderhane L, Reis AF, Hebling J, Mazzoni A, Breschi L, et al. In vivo preservation of the hybrid layer by chlorhexidine. J Dent Res. 2007;86(6):529-33.

26. Hardan L, Bourgi R, Kharouf N, Mancino D, Zarow M, Jakubowicz N, Haikel Y, Cuevas-Suárez CE. Bond strength of universal adhesives to dentin: a systematic review and meta-analysis. Polymers (Basel). 2021;13(5):814.

27. Haak R, Siegner J, Ziebolz D, Blunck U, Fischer S, Hajtó J, Frankenberger $\mathrm{R}$, Krause F, Schneider H. OCT evaluation of the internal adaptation of ceramic veneers depending on preparation design and ceramic thickness. Dent Mater. 2021;37(3):423-31.

28. Blunck U, Fischer S, Hajtó J, Frei S, Frankenberger R. Ceramic laminate veneers: effect of preparation design and ceramic thickness on fracture resistance and marginal quality in vitro. Clin Oral Invest. 2020:24(8):2745-54

29. Pekkan G, Hekimoglu C. Evaluation of shear and tensile bond strength between dentin and ceramics using dual-polymerizing resin cements. J Prosthet Dent. 2009;102(4):242-52.

30. Özcan M, Pekkan G. Effect of different adhesion strategies on bond strength of resin composite to composite-dentin complex. Oper Dent. 2013;38(1):63-72.

31. Abdelraouf RM, Mohammed M, Abdelgawad F. Evaluation of shear-bondstrength of dental self-adhering flowable resin-composite versus totaletch one to enamel and dentin surfaces: an in-vitro study. Open Access Maced J Med Sci. 2019;7(13):2162-6.

32. Atabek \$̆, Özden AN. Comparison of the effect of proanthocyanidin surface treatments on shear bond strength of different cements. Materials (Basel). 2019:12(17):2676.

33. Bulut AC, Atsü SS. The effect of repeated bonding on the shear bond strength of different resin cements to enamel and dentin. J Adv Prosthodont. 2017:9(1):57-66.

34. Koodaryan R, Hafezeqoran A, Khakpour Maleki A. The effect of resin cement type and cleaning method on the shear bond strength of resin cements for recementing restorations. J Adv Prosthodont. 2017:9(2):110-7.

35. Chu SJ, Mieleszko AJ. Color matching strategies for non-vital discolored teeth, part 2: in-vivo bleaching options for discolored teeth preparations. J Esthet Restor Dent. 2015;27(Suppl 1):S18-23.

36. Abanto Alvarez J, Rezende KM, Marocho SM, Alves FB, Celiberti P, Ciamponi AL. Dental fluorosis: exposure, prevention and management. Med Oral Patol Oral Cir Bucal. 2009;14(2):E103-107.

37. Edelhoff $D$, Brix $O$. All-ceramic restorations in different indications: a case series. J Am Dent Assoc. 1939;2011(142 Suppl 2):14s-9s.

38. Ibarra G, Johnson GH, Geurtsen W, Vargas MA. Microleakage of porcelain veneer restorations bonded to enamel and dentin with a new selfadhesive resin-based dental cement. Dent Mater. 2007:23(2):218-25.

39. Petridis HP, Zekeridou A, Malliari M, Tortopidis D, Koidis P. Survival of ceramic veneers made of different materials after a minimum follow-up period of five years: a systematic review and meta-analysis. Eur J Esthet Dent. 2012;7(2):138-52.

\section{Publisher's Note}

Springer Nature remains neutral with regard to jurisdictional claims in published maps and institutional affiliations.
Ready to submit your research? Choose BMC and benefit from:

- fast, convenient online submission

- thorough peer review by experienced researchers in your field

- rapid publication on acceptance

- support for research data, including large and complex data types

- gold Open Access which fosters wider collaboration and increased citations

- maximum visibility for your research: over $100 \mathrm{M}$ website views per year

At BMC, research is always in progress.

Learn more biomedcentral.com/submissions 